\title{
RF COMPONENTS USING OVER-MODED RECTANGULAR WAVEGUIDES FOR THE NEXT LINEAR COLLIDER MULTI-MODED DELAY LINE RF DISTRIBUTION SYSTEM *
}

\author{
S. G. Tantawi $^{\# \$}$, N. M. Kroll, And K. Fant, SLAC, Stanford, CA
}

\begin{abstract}
We present the design and analysis for a set of smooth transitions from rectangular to circular waveguide that preserves their common reflection symmetries. The Smatrix of the transition connects modes of the same symmetry class, and for a sufficiently adiabatic transition preserves their TE (or TM) character. It is then also nonreflecting and, in the absence of degeneracy, its modal connections are one to one and order preserving. This property enables us to carry out all of the RF manipulations in the more easily handled over-moded rectangular waveguide.
\end{abstract}

\section{INTRODUCTION}

The Multi-moded Delay Line Distribution System (MDLDS) was suggested as an alternative for rf pulse compression for the Next Linear Collider (NLC)[1-2]. MDLDS propagates several modes in a single circular highly over-moded waveguide. This system uses a set of complicated over moded rf components. Most of these components manipulate several modes at the same time.

Manipulation of several modes in a single component is easier in rectangular waveguides. To take advantage of this, we present several rf tapers which maps the modes in circular waveguides into modes in rectangular guides. Three types of tapers are presented:

1. A taper from circular waveguide to a square waveguide. The circular guide diameter is such that all modes with cut-off frequency above that of the $\mathrm{TE}_{01}$ do not propagate.

2. A taper from circular waveguide to a square waveguide with the circular waveguide diameter is such that all modes with cut-off frequency above that of the $\mathrm{TE}_{12}$ do not propagate.

3. A taper from circular waveguide to a rectangular guide. The circular guide diameter is such that all modes with cut-off frequency above that of the $\mathrm{TE}_{01}$ do not propagate.

"This work is supported by Department of Energy Contract DE-AC0376 SF00515.

\#Email: tantawi@slac.stanford.edu

${ }^{\text {s} A l s o ~ w i t h ~ t h e ~ C o m m u n i c a t i o n s ~ a n d ~ E l e c t r o n i c s ~ D e p a r t m e n t, ~ C a i r o ~}$ University, Giza, Egypt.

\section{SIMULATION TECHNIQUE}

We assumed that all these tapers will be built using wire Electron Discharge Machining (EDM). When tapering from one shape, e.g. a circle, to another shape, e.g., a square, the length of the taper $l$ and the connecting points between the two shapes uniquely define the taper. In cylindrical coordinates a shape $i$ placed with cylindrical symmetry around the z-axis can be described by a relation $r_{i}(\phi)$, which gives the radius as a function of the angle $\phi$. The taper between two shapes $r_{1}(\phi)$, and $r_{2}(\phi)$ is then given by

$$
r(\phi, z)=r_{1}(\phi)+\frac{r_{2}(\phi)-r_{1}(\phi)}{l} z .
$$

This taper is compatible with the process of wire EDM when the two heads of the machine are moving synchronously with the same angular speed. More complicated tapers are described by a set of tapers, each have the form of Eq.(1) and cascaded together.

All simulations were performed using HFSS [3]. The shapes $r_{i}(\phi)$ were plotted using a finite number of points (72 points) using Visual Basic Application (VBA) in AutoCAD [4]. The shapes were then imported into HFSS, and the interpolation described in Eq. (1) was done using the connect function, to generate the solid model. This process was used to design all the tapers described in this work. All tapers were optimized around $11.424 \mathrm{GHz}$.

\section{TAPER DESIGNS}

\subsection{Type 1: $T E_{01}$ Circular to Square Taper}

In this taper the circular waveguide diameter is chosen such that all modes, that have a cut-off frequency above that of the $\mathrm{TE}_{01}$ mode do not propagate. The square waveguide is just large enough to allow both $\mathrm{TE}_{20}$ and $\mathrm{TE}_{02}$. However, it does not allow the propagation of $\mathrm{TE}_{22}$ and $\mathrm{TM}_{22}$ modes. Because of reflection symmetries, only the two degenerate modes, $\mathrm{TE}_{20}$ and $\mathrm{TE}_{02}$, in the rectangular guide are exited when the incident mode in the circular guide is $\mathrm{TE}_{01}$. The design process for this taper is simply done by increasing the length until the reflection coefficient for the $\mathrm{TE}_{01}$ mode in the circular guide is small enough. Fig. 1 shows the taper design and Fig. 2 shows the $\mathrm{TE}_{01}$ mode reflection coefficient for this design. Because of degeneracy the combination between the two modes in the square waveguide could be regarded as one single 
mode. When exciting the circular guide with the $\mathrm{TE}_{12}$ mode, again, it couples to the two modes $\mathrm{TE}_{02}$ and $\mathrm{TE}_{20}$. However, the phase between them is a 180-degree different from the previous case, i.e., when they are excited because of the $\mathrm{TE}_{01}$ mode in the circular guide. Again this combination could be regarded as a different single mode in the square guide.

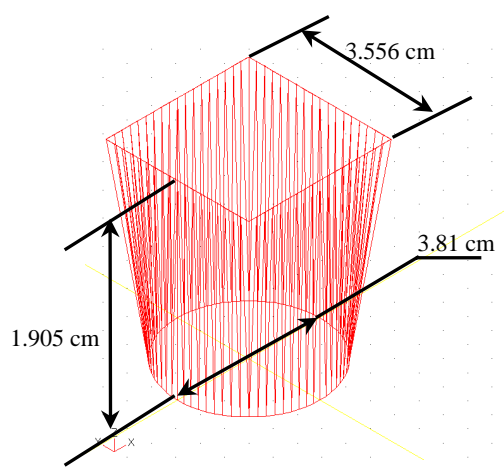

Fig. 1 Type 1 taper design

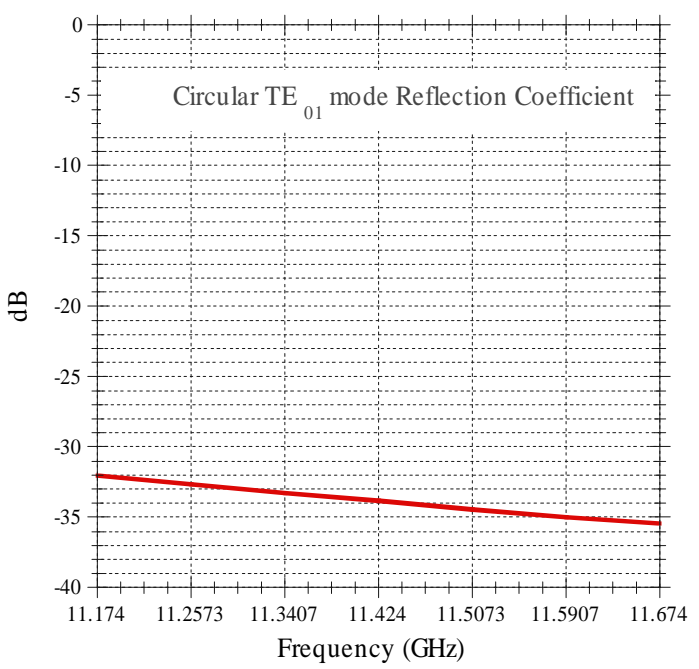

Fig. 2. Simulated results of the type 1 taper design.

\subsection{Type 2: $T E_{01}$ and $T E_{12}$ Circular to Square Taper.}

The circular waveguides used in the long delay lines of the MDLDS uses three different modes. These modes are the $\mathrm{TE}_{01}$, and the two polarizations of the $\mathrm{TE}_{12}$ mode [1]. In tapers of this type, the diameter of the circular waveguide is increased to $5.08 \mathrm{~cm}$ to support the $\mathrm{TE}_{12}$ mode. Correspondingly, the width of the square waveguide was increased to $4.491 \mathrm{~cm}$ to support the $\mathrm{TE}_{30}$ and the $\mathrm{TE}_{03}$ modes. At this width the square waveguide supports both the $\mathrm{TE}_{22}$ and the $\mathrm{TM}_{22}$ modes,. These modes have the same reflection symmetries as the $\mathrm{TE}_{01}$ mode in circular waveguide and the $\mathrm{TE}_{02}$ and $\mathrm{TE}_{20}$ in rectangular waveguide. The design process of this taper was simply increasing the length until the coupling from the circular $\mathrm{TE}_{01}$ mode to the square $\mathrm{TE}_{22}$ and $\mathrm{TM}_{22}$ is small enough.

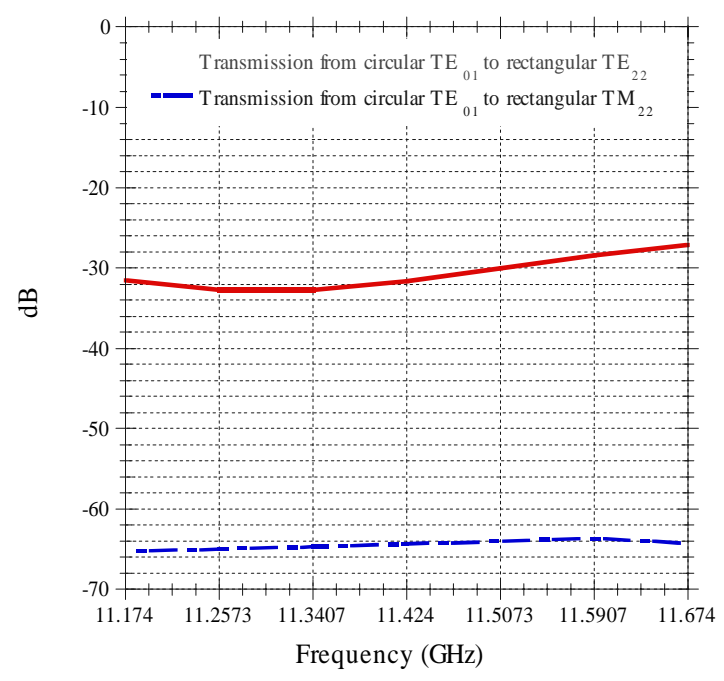

Fig. 3 coupling of the $\mathrm{TE}_{01}$ in the circular guide to spurious modes in the square guide. At the design length for Type 2 taper.

The length required is $10.16 \mathrm{~cm}$. Fig. 3 shows the coupling of the $\mathrm{TE}_{01}$ in the circular guide to unwanted modes in the square guide. At this length the coupling between modes in the circular guide to modes in the square guide is one to one with a transmission coefficient that is better than $-0.017 \mathrm{~dB}$ over a $0.5 \mathrm{GHz}$ bandwidth around $11.424 \mathrm{GHz}$. The modes connect to each other according to the following table:

\begin{tabular}{|c|c|}
\hline Circular Guide modes & Square Guide Modes \\
\hline TE11 (Polarization \#1) & TE10 \\
\hline TE11(Ploarization \#2) & TE01 \\
\hline TM01 & TM11 \\
\hline TE21 (Polarization \#1) & $\begin{array}{c}\text { TE20 and TE02 (In } \\
\text { Phase) }\end{array}$ \\
\hline TE21 (Polarization \#2) & TE11 \\
\hline TE01 & $\begin{array}{c}\text { TE20 and TE02 (out of } \\
\text { Phase by 180 degrees) }\end{array}$ \\
\hline TM11 (Polarization\#1) & TM12 \\
\hline TM11 (Polarization\#2) & TM21 \\
\hline TE31 (Polarization \#1) & TE12 \\
\hline TE31 (Polarization \#2) & TE21 \\
\hline TM21 (Polarization \#1) & TM22 \\
\hline TM21 (Polarization\#2) & TM13 and TM13 (In \\
& phase) \\
\hline TE41 (Polarization\#1) & TE22 \\
\hline TE41 (Polarization\#2) & TE31 and TE13 \\
\hline TE12(Polarization\#1) & TE30 \\
\hline TE12(Polarization\#1) & TE03 \\
\hline TM02 & $\begin{array}{c}\text { TM31 and TM13 (out of } \\
\text { phase by 180 degrees) }\end{array}$ \\
\hline
\end{tabular}




\subsection{Type 3: $T E_{01}$ Circular to $T E_{02}$ Rectangular Taper.}

In several applications one would want to convert the TE01 mode in the circular guide to a single polarization of the $\mathrm{TE}_{02}$ in the square guide. Modifying the square waveguide to a rectangular waveguide to break the degeneracy between the TE02 and TE20 modes could do this. However, in this case, the length of the taper required to achieve an adiabatic transition to a single mode in the rectangular guide is excessive (approximately $17.78 \mathrm{~cm}$ ). Instead, we construct this taper from three sections as shown in Fig 4.

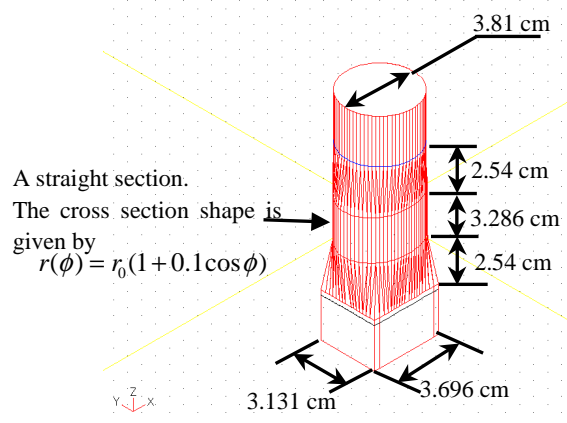

Fig. 4. The design of a $\mathrm{TE}_{01}$ Circular to $\mathrm{TE}_{02}$ Rectangular Taper.

The middle section is a cylinder with the following shape

$$
r_{2}(\phi)=r_{0}(1+0.1 \cos 2 \phi)
$$

where $r_{0}$ is the radius of the circular guide. The dimensions of the rectangular guide is chosen such that the rectangular cross section satisfies the following equation

$$
r_{3}(\phi)=r_{0}\left(1+0.1 \cos \phi+\sum_{i=2}^{\infty} \alpha_{i} \cos 2 i \phi\right)
$$

where the coefficients $\alpha_{i}$ are chosen to produce a rectangular shape.

The taper from the circle to the intermediate shape scatters the $\mathrm{TE}_{01}$ mode into two modes $\mathrm{M}_{1}$ and $\mathrm{M}_{2}$ in the intermediate section. Also the taper between the rectangular waveguide to the intermediate shape scatters the rectangular mode $\mathrm{TE}_{02}$ into $\mathrm{M}_{1}$ and $\mathrm{M}_{2}$. The lengths of both tapers are adjusted such that the magnitude of the coefficients of the scattered modes $M_{1}$ and $M_{2}$ are the same from both sides. Since $\mathbf{M}_{1}$ and $\mathbf{M}_{2}$ propagate with deferent phase velocities in the intermediate section, the length of that section could be adjusted so that the circular $\mathrm{TE}_{01}$ mode get completely converted into the rectangular $\mathrm{TE}_{02}$ mode.

Fig. 5 shows the simulated performance of that taper. The coupling to the cross-polarized mode is below $20 \mathrm{~dB}$. Further refinement of the design could be made to reduce this level further.

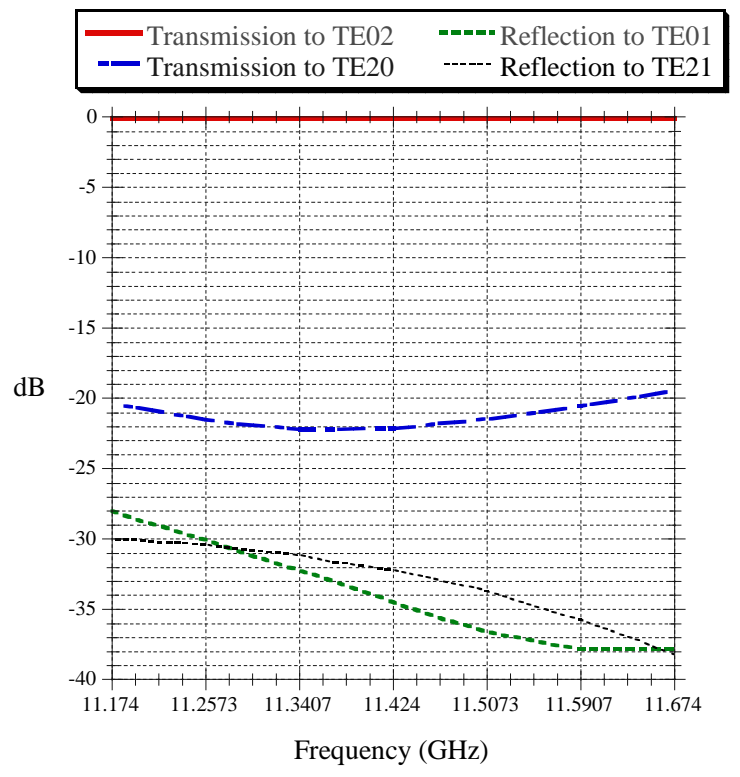

Fig. 5 Simulated performance of type 3 taper

\section{CONCLUSION}

We presented a set of smooth transitions from rectangular to circular waveguide. These transition maps modes from circular guides into modes in rectangular guides with one to one model connection. We showed that these tapers could be quite compact and efficient. These tapers could be used in a variety of applications for designing over-moded microwave components. All rf manipulations could be made in the more easily handled rectangular waveguide, while tapering back and forth to circular waveguide.

\section{ACKNOWLEDGMENT}

The authors wish to thank Z. D. Farkas, P.B. Wilson, R. M. Miller and R. D. Ruth for many useful discussions.

\section{REFERENCES}

[1] The NLC Design Group "Zeroth-Order Design Report for the Next Linear Collider," SLAC-Report474, May 1996.

[2] S.G. Tantawi, et al. " A Multi-Moded RF Delay Line Distribution System for the Next Linear Collider," to be published in the proc. of the Eighth Workshop on Advanced Accelerator Concepts, Baltimore, MD, USA 6-11 Jul 1998.

[3] "HP High-Frequency Structure Simulator," HP EEsof, Hewalt Packard.

[4] AutoCAD, Copyright 1982-1998, Autodesk, Inc. 